\title{
Research Paper: Motor Speech Sequence Learning in Adults Who Stutter
}

\author{
Mahsa Aghazamani ${ }^{1},{ }^{*}$ Mohammad Rahim Shahbodaghi ${ }^{1}$, Elham Faghihzadeh ${ }^{2}$
}

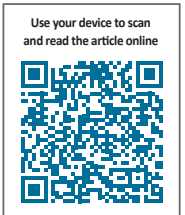

Cittat on: Aghazamani M, Shahbodaghi MR, Faghihzadeh E. [Motor Speech Sequence Learning in Adults Who Stutter (Persian)]. Archives of Rehabilitation. 2018; 19(1):26-35. https://doi.org/10.21859/JREHAB.19.1.26

https://doi.org/10.21859/JREHAB.19.1.26

Received: 24 Aug. 2017

Accepted: 01 Jan. 2018

\begin{abstract}
Objective Developmental stuttering is a speech disorder characterized by repetition, prolongation, block and disruption of the smooth flow of speech. Environmental, physical, mental, and cognitive-linguistic factors were involved in the initiation and development of stuttering. There have been several theories about the development of stuttering. One of these theories suggests that stuttering is a speech motor control disorder. Based on the speech-motor skills hypothesis, speech production is a motor skill similar to any other (fine) motor skill that humans possess, and the individual motor skills develop from the lowest skill level to the highest level of motor skills. The SMS view suggests that stuttering may arise from limitations in speech motor skill in people who stutter are located more toward the lower end of a presumed normal speech motor skill continuum. Therefore, people who stutter have difficulty learning speech motor skills and have a limited ability to benefit from speech motor practice compared to normal individuals. Many of the stuttering treatment programs teach the new pattern of speech, and the success of these treatment programs depends on the learning of the new speech patterns. So if people who stutter have limited speech motor skills, also will have little success in this treatment programs. Therefore, this study aimed to investigate motor speech sequence learning in adults who stutter.

Materials \& Methods This was a descriptive-analytical study with participants as 15 adults who stutter and 15 adults who do not stutter. All subjects were matched for age, sex and educational level. The average age was 28.73 for the adults who stutter was 28.4 for the normal people. All of the participants had history of neurological and motor speech disorders. The convenience sampling method was used for this study. The stuttering severity of people who stutter was assessed with Wingate scale and was in moderate range. Participants were asked to repeat an eight syllable non word sequence 30 times during three practice sessions. Recorded samples were analyzed with PRAAT software, and the variables used to measure performance gains included accuracy, response preparation time and sequence duration. The data were statistically analyzed by SPSS software (version 23).

Results Based on the findings of the present study, PWS showed improvement in accuracy from day 1 to day $3(P<0.05)$, but there was no difference between the two groups in this variable $(P>0.05)$. Also, the reaction time of the PWS improved from day 1 to day $3(P<0.05)$, but there was no difference between the two groups $(P>0.05)$. The sequence duration of the PWS improved from day 1 to day $3(P<0.05)$ and there was a significant difference between the two groups $(P<0.05)$. According to the results of this study, PWS showed slower sequence duration compare to PNS.

Conclusion The results of this study showed that PWS show improvement in accuracy, reaction time and sequence duration variables from day 1 to day 3 . Also, PWS show more substantial number of errors compared to PNS, but this difference was not significant between the two groups. Similar results were obtained for the reaction time. Results of this study demonstrated that PWS show slower sequence duration compared to PNS. Some studies suggested that this could be because people who stutter use a control strategy to reduce the number of errors, although many studies suggested that this may indicate motor learning. According to speech motor skills hypothesis, it can be concluded that people who stutter have limitations in motor speech learning abilities. The findings of the present study could have clinical implication for the treatment of stuttering.
\end{abstract}

Keywords: Developmental stuttering, Adult, Learning, Motor, Speech

\section{* Corresponding Author:}

Mohammad Rahim Shahbodaghi, MSc.

Address: Department of Speech Therapy, School of Rehabilitation, Tehran University of Medical Sciences, Tehran, Iran.

Tel: +98 (21) 22228051

E-Mail: shahbodaghi@sina.tums.ac.ir 


\title{
يادتيرى توالىهاى حركتى كَفتار در بزرتىسالان مبتلابه لكنت
}

\author{
مهسا آقازمانى'، "محمدرحيم شاهبداغى' 'ه، الهام فقيدزاده'

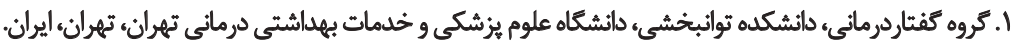

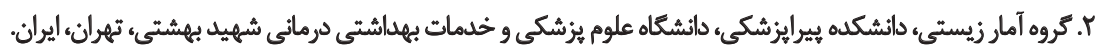

\begin{abstract}
حكיد

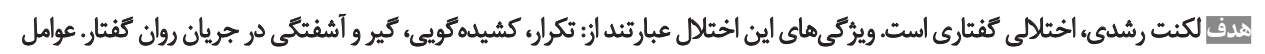

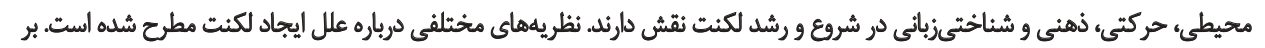

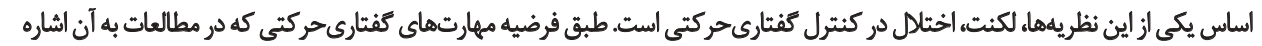

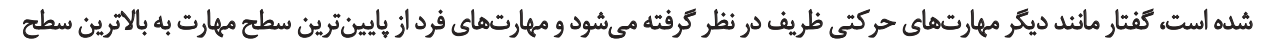

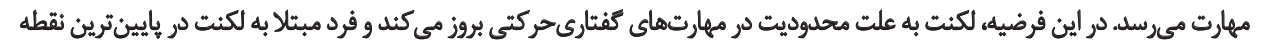

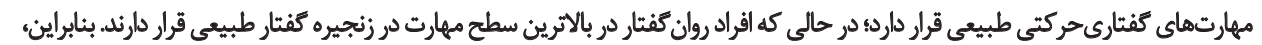

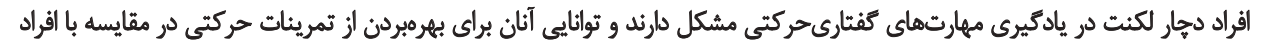

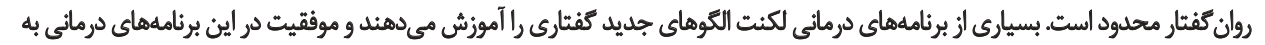

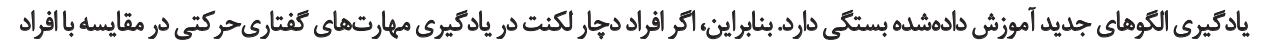

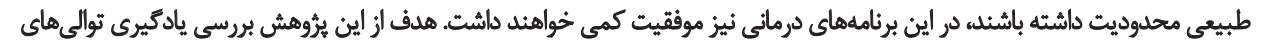

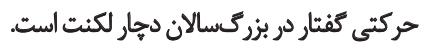

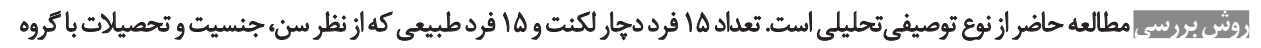

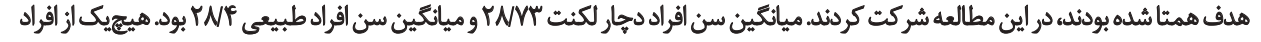

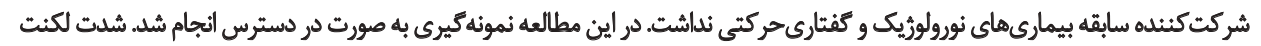

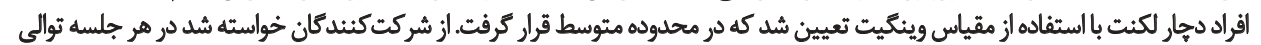

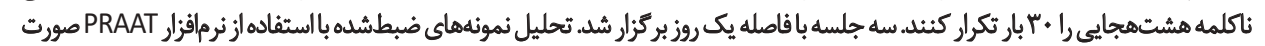

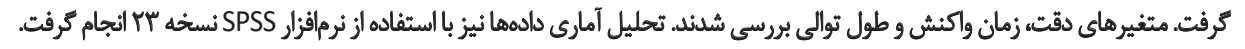

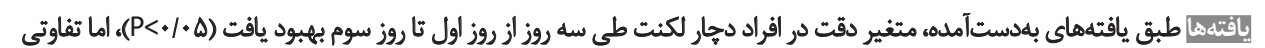

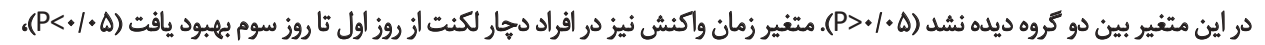

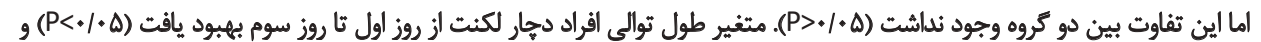

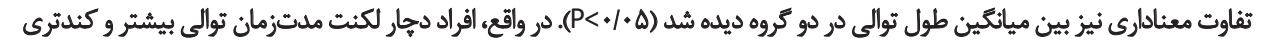
در مقايسه با افراد طبيعى داشتيند

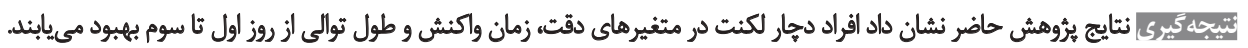

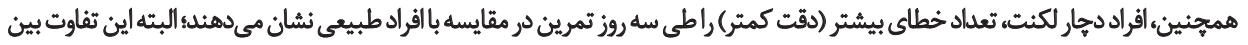

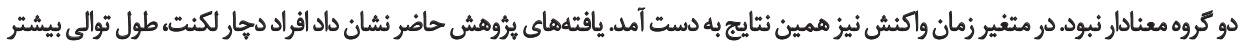

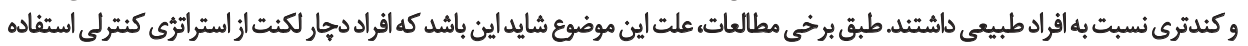

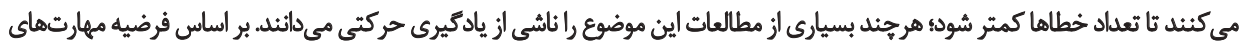

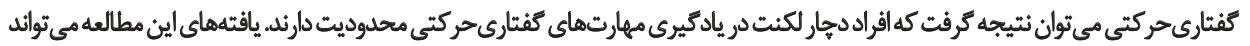

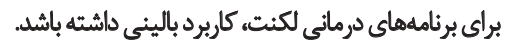

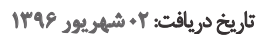

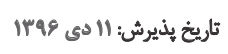


تكليفى جديد يا حفظ عملكرد به دنبال دورهاى از استراحت

مقلمه

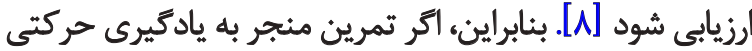

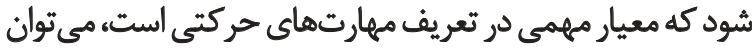

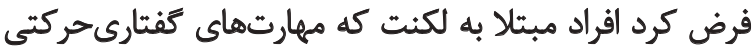

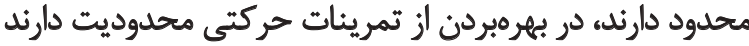

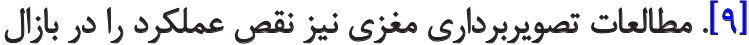

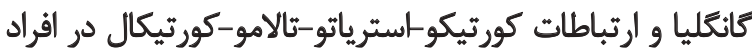

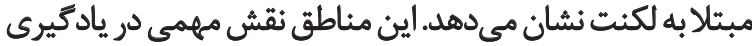
توالىهاي حركتى و افزرايش خودكارسازي با تمرين دارند [ـ [1].

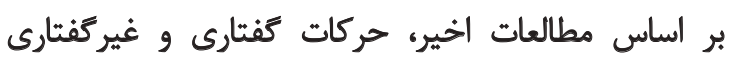

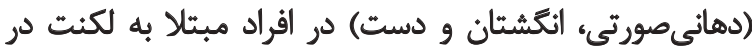

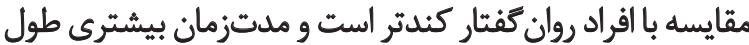

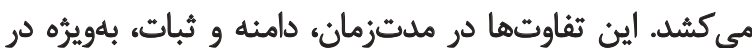

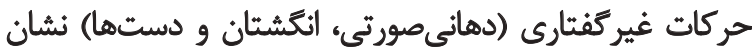

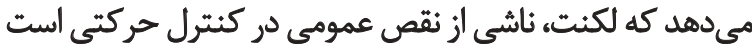

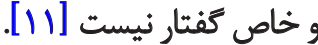
مطالعاتى كه توانايى كمتر افراد مبتلا به لكثت رادر يادئ يادكيرى

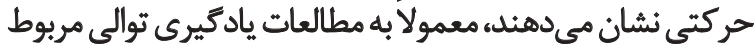

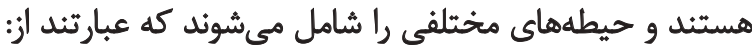

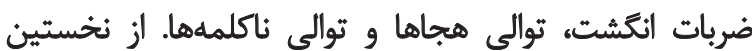

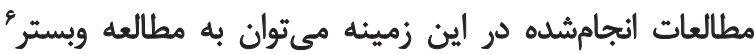

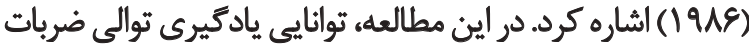

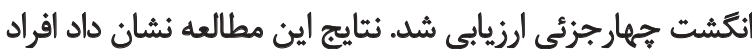

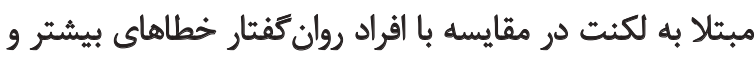
زمان واكنش طولانى ترى با تمرين دارند [IT)

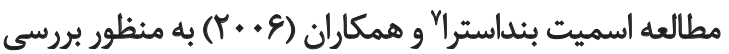

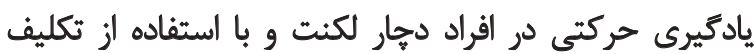

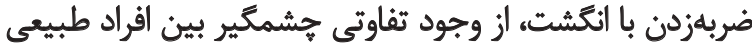

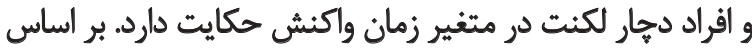

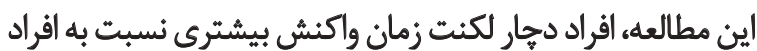

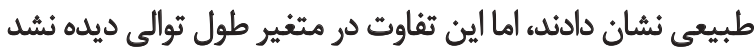

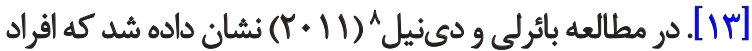

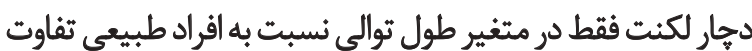

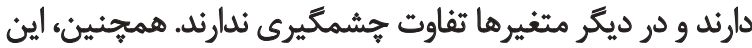

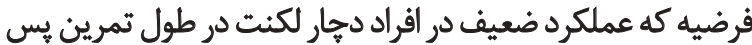

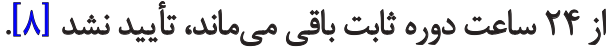

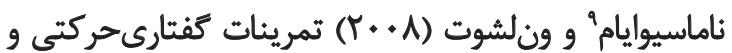

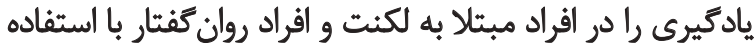

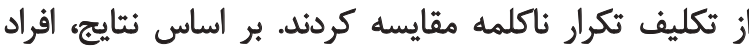

\section{Webster}

7. Smits-Bandstra

8. Bauerly \& De Nil

9. Namasivayam
الكنت رشدى، اختلالى كفتارى است. ويرّكى هاى اين اختلال

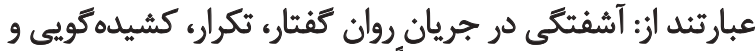

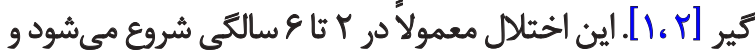

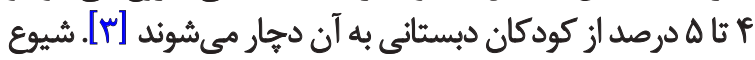

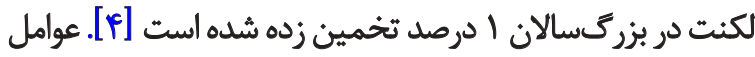

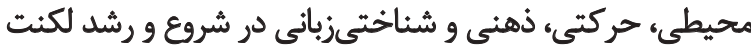

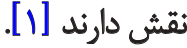

نظريههاي مختلفى درباره علل ايجاد لكنت مطرح شده است

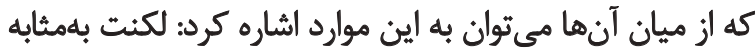

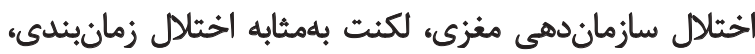

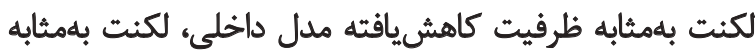

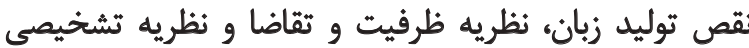

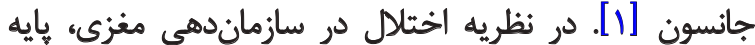

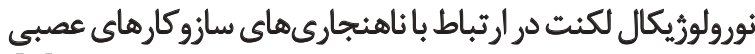

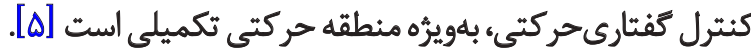

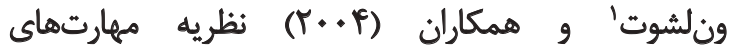

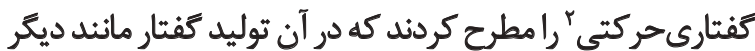

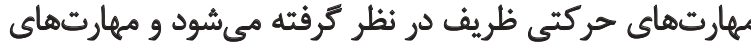

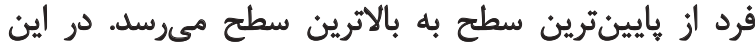

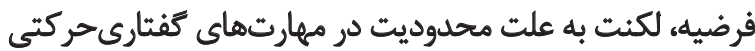

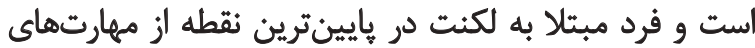

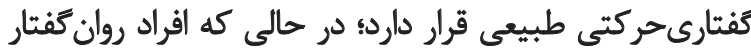

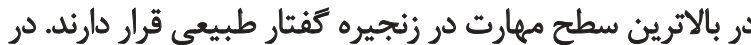

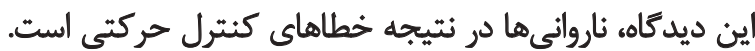

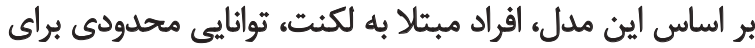

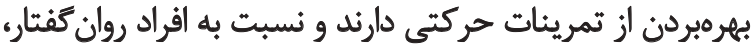

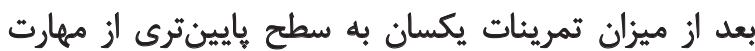
حركتى مىرسند [عان مينات طبق مشاهدات، يادكيرى حركات كفتارى ذاتى نيست و و

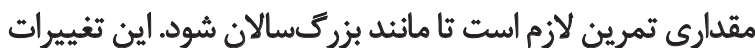

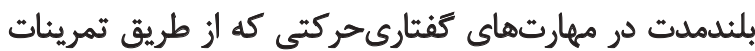
تكرارشونده به دست مى آيند، نشانديندي

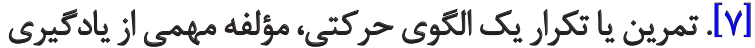

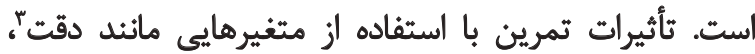

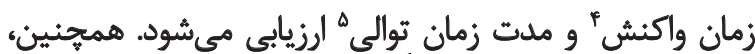

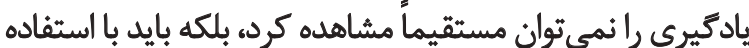

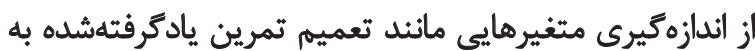

1. Van Lieshout

2. Speech Motor Skills (SMS)

3. Accuracy

4. Reaction time

5. Sequence duration 


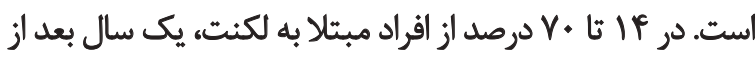

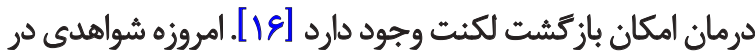

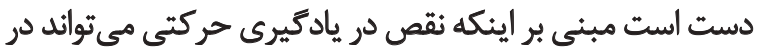

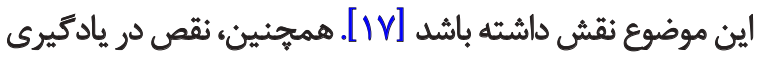

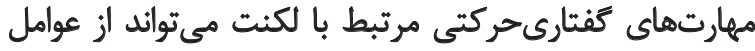

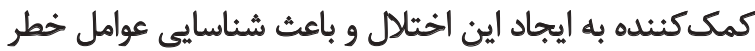

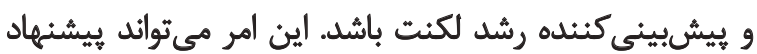
تصحيح روشهاي درمانى لكنت را مطرح كند.

با توجه به تفاوتها و تناقض هاى مشاهدهشده در نتايج مطالعات

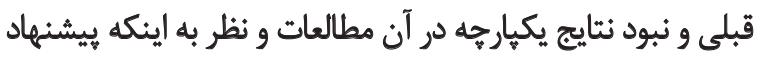

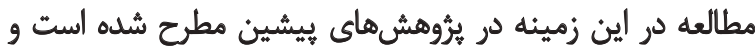

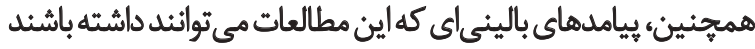

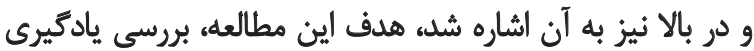

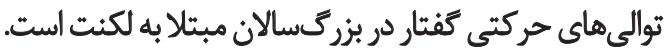

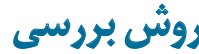

اين مطالعه از نوع توصيفىتحليلى است. نمونهاي اين

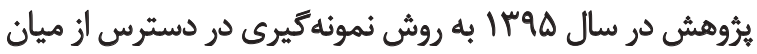

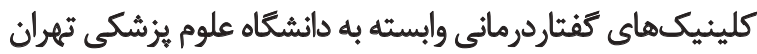

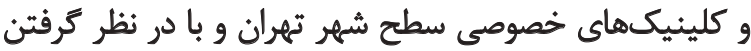

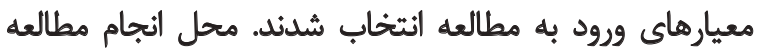

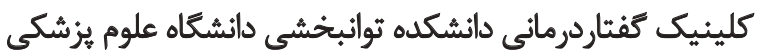

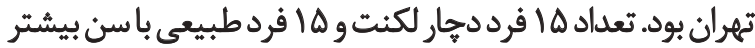

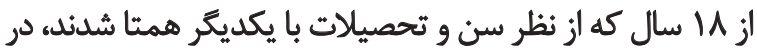

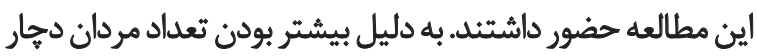

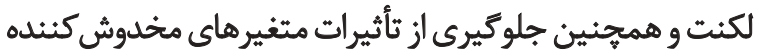

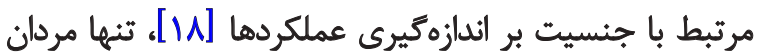

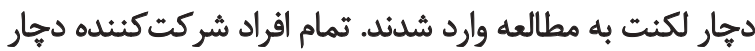

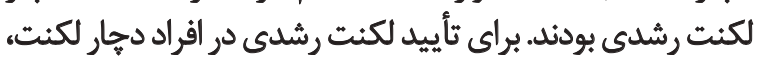

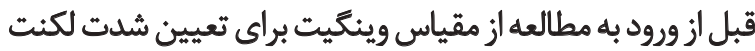

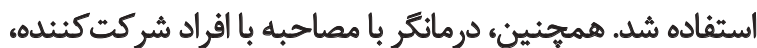
اين موضوع را تأييد كرد.

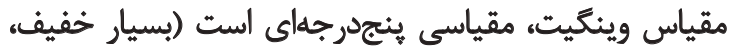

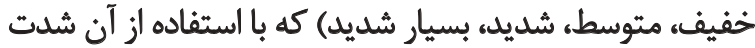

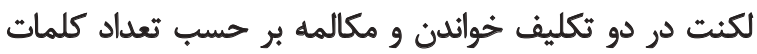

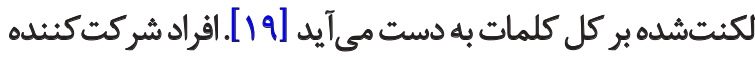

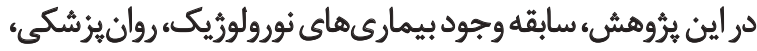

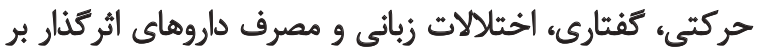

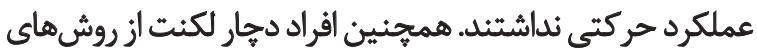

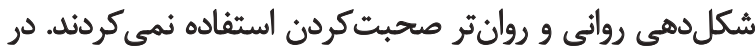

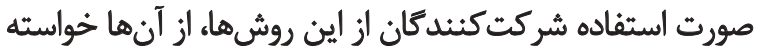

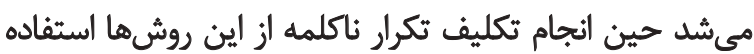

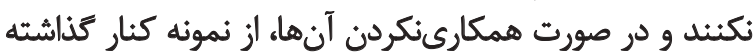

دهار لكنت و افراد روان كَفتار مي توانئد در بسيارى از متغيرهاي

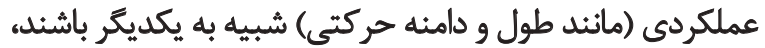

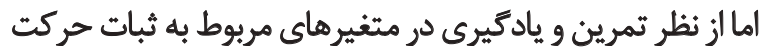

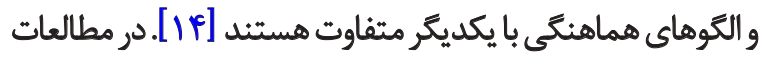

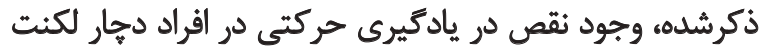

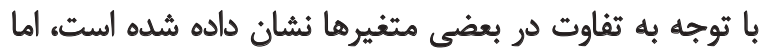

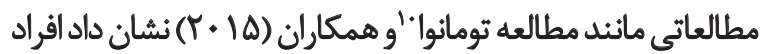

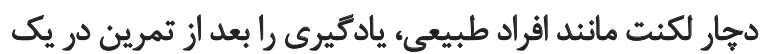

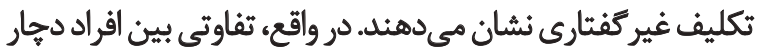

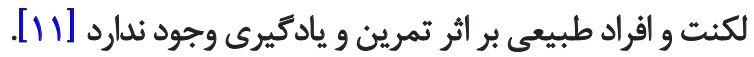
مطالعات مختلف نتايج متفاوتى را با توجه به متغيرهايى

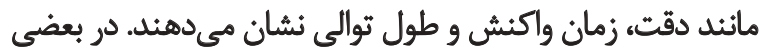

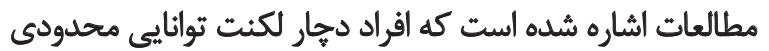

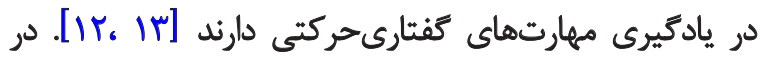

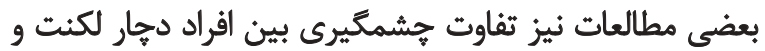

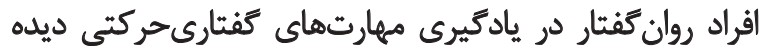

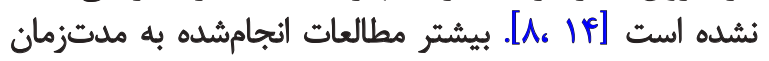

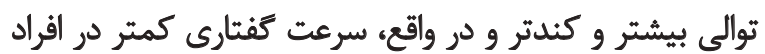

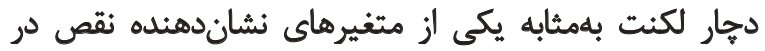

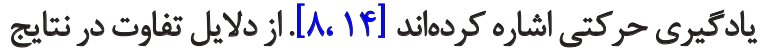

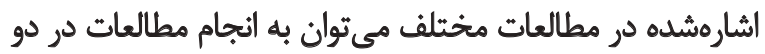

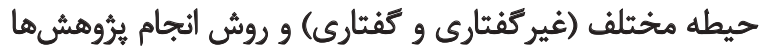

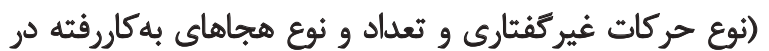
تمرين) اشاره كرد.

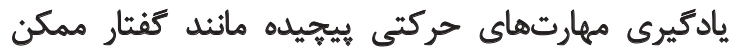

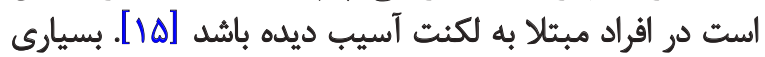

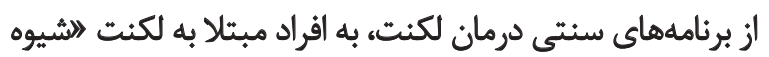

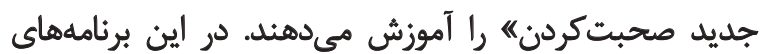

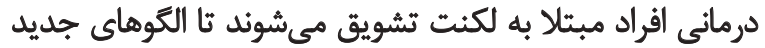

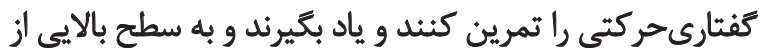

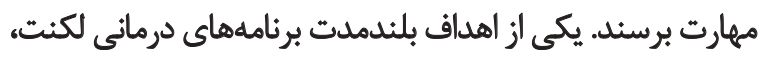

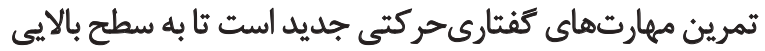

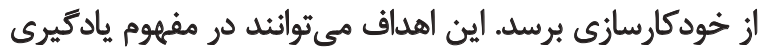

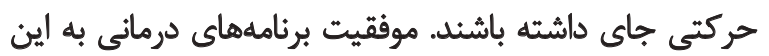

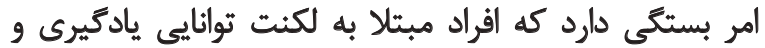

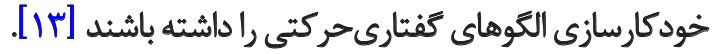
با توجه به اينكه اهداف بلندمدت برئامهنهاي روائي، دستيابي

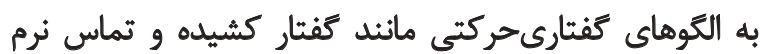

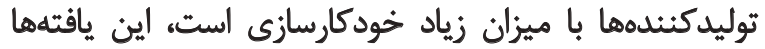

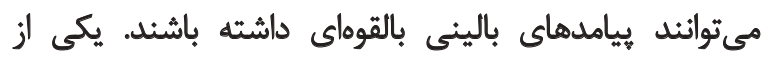

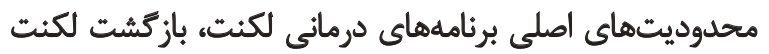

10. Tumanova 


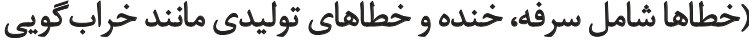

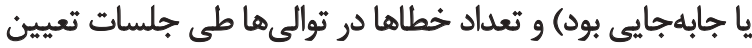

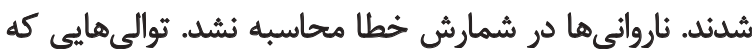

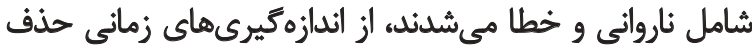

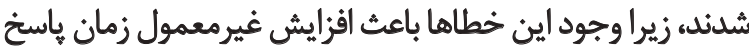

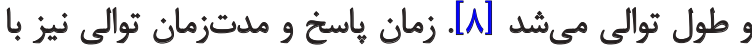

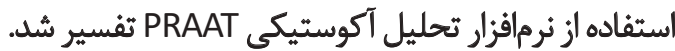

اندازهيرى زمان هاسخ به اين صورت بود كه از زمان آغاز

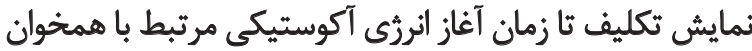

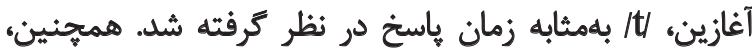

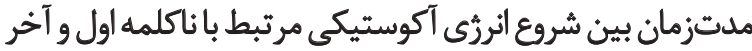

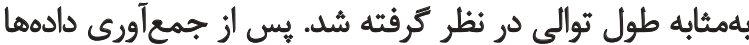

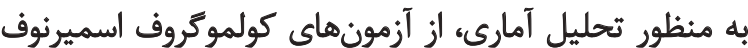

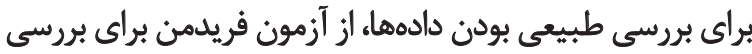

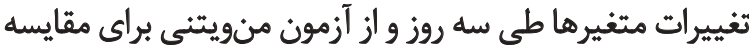

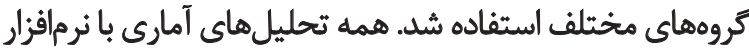

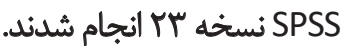

يافتهها

در مطالعه حاضر، 10 فرد بزركسال دجار لكنت با ميانكين

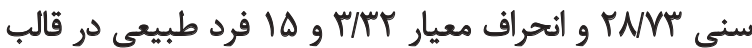

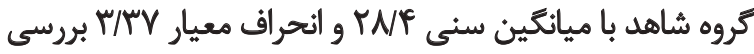

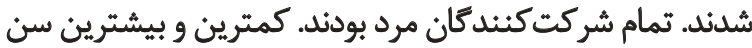

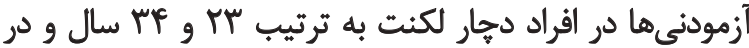

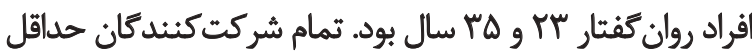

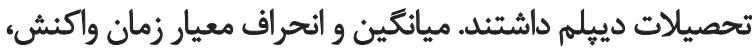

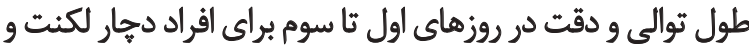
كروه شاهد در جدول شماره إنشان داده شده است.

از آنجا كه نتايج آزمون كولموكروف اسميرنوف طبيعى بودن

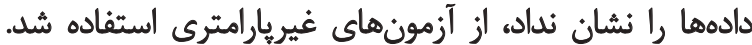

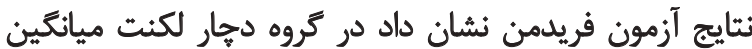

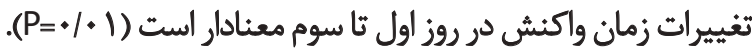

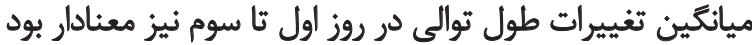

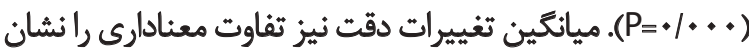

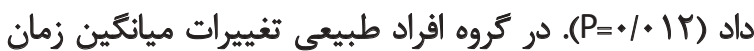

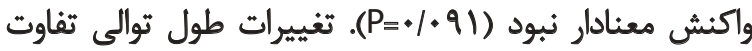

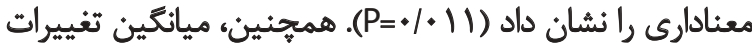

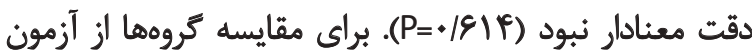

$$
\text { منويتنى استفاده شد. }
$$

مقدار P براى مقايسه دو كروه در مؤلفههاى زمان واكتش، طول

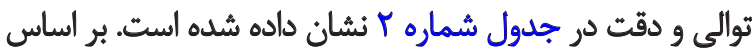

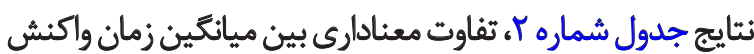

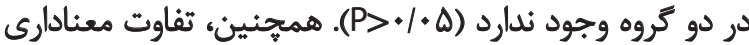

مى شدندء زيرا روش هاى درمانى كه عموماً شامل شكل دهاهى روانى

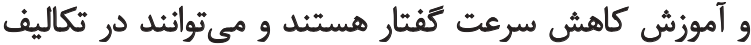

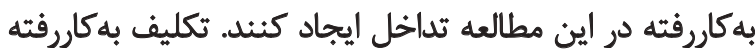

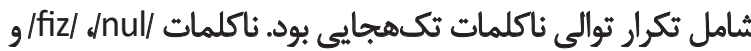
/tux/،dač/lgeŠ/

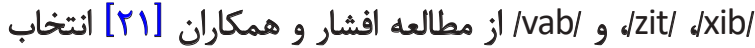

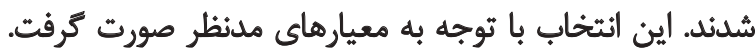

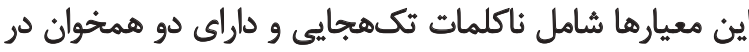

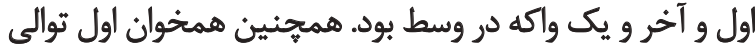

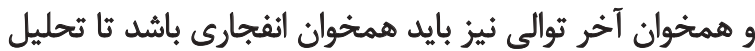

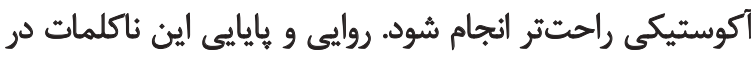

$$
\text { ئروهشهاى مذكور تعيين شده است. }
$$

سرانجام، 1 ناكلمه تكهجايي در كنار هم قرار داده شداد

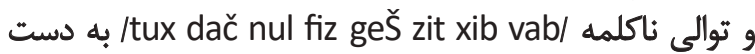

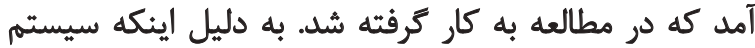

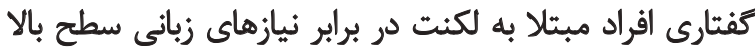

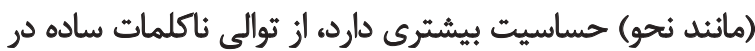

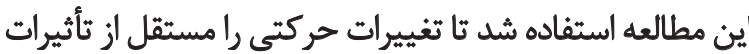

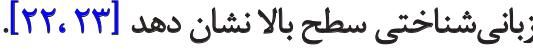

در اين مطالعه، سه جلسه با فاصله زمانى هر جلسه يك ئ روز

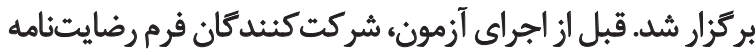

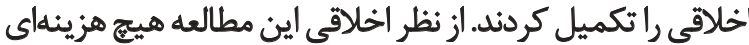

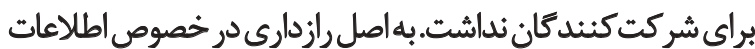

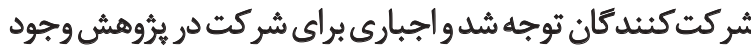

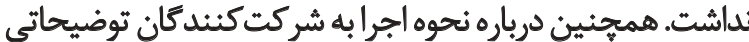

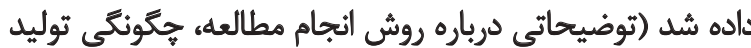

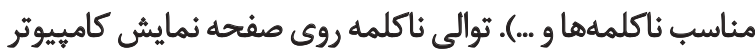

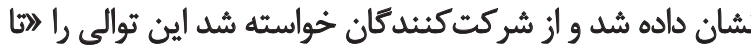

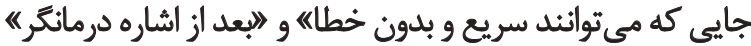

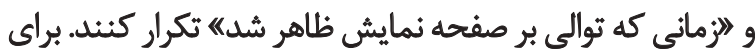

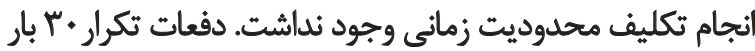

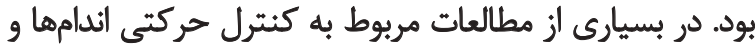

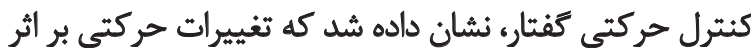

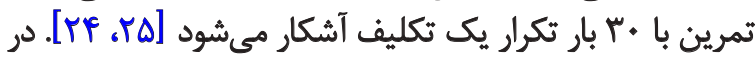

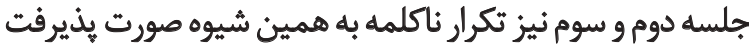

و تمام جلسات به صورت صوتى و تصويرى ضبط شئ شدند. با توجه به اينكه متغيرهاي نشاندهنده يادكيرى حركتى دقت.

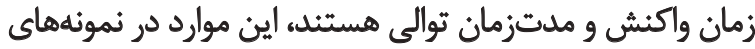

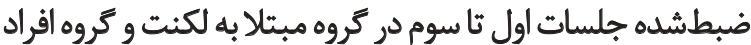

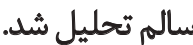

براى تعيين دقت، تمام دادها در هر دو كروه بر اساس ضبط

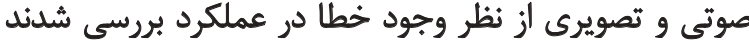


جدول ا. ميانكين و انحراف معيار زمان واكثش، طول توالى و دقت در افراد دهار لكنت و افراد طبيعى

\begin{tabular}{|c|c|c|c|}
\hline انحراف معيار & مياتكين & متغيرها & \\
\hline .194 & $.19 T$ & زمان واكنش ال(بر حسب ثائيه) & \multirow{9}{*}{ كروه دهار لكنت } \\
\hline$\cdot M$ & $\cdot / r$ & زمان واكنش r (بر حسب ثائيه) & \\
\hline .110 & $\cdot / \pi$ & زمان واكش ب (بر حسب ثائيه) & \\
\hline $\mathbb{M}$ & $m$ & طول توالى ( (بر حسب ثانيه) & \\
\hline. $\operatorname{lex}$ & r/AV & طول توالئ Y (ير حسب ثانيه) & \\
\hline .108 & $r / \varepsilon$ & طول توالى بّ (بر حسب ثاثيه) & \\
\hline IF/AY & NIT & دقت & \\
\hline QNO & $\Delta / N$ & دقت ץ & \\
\hline$\Delta / Q V$ & $r / A$ & دقت & \\
\hline .100 &.$/ P V$ & زمان واكنش ا (بر حسب ثاثيه) & \multirow{9}{*}{ كروه طبيعى } \\
\hline-119 &.$/ \pi$ & زمان واكنش r (بر حسب ثائيه) & \\
\hline.$/ 18$ & $\cdot / \pi$ & زمان واكثش ب (بر حسب ثائيه) & \\
\hline .ND & r/gT & طول توالى ا (بر حسب ثانيه) & \\
\hline$\cdot M$ & r/rq & طول توالئ Y (إبر حسب ثانيه) & \\
\hline.$/ 4 \Delta$ & $r M I$ & طول توالى ب (بر حسب ثاثيه) & \\
\hline$m / 1$ & $r / \pi$ & دقت ا & \\
\hline r/q9 & $r$ & دقت & \\
\hline $\mathbb{M}$ & $1 / r$ & دقت ب & \\
\hline
\end{tabular}

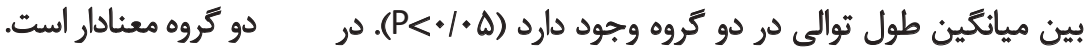

در اين مطالعه، افراد دجار لكنت ميانكين تعداد خطاي بيشترى

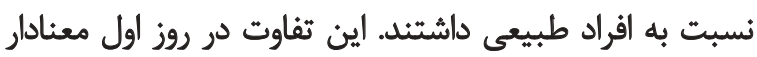

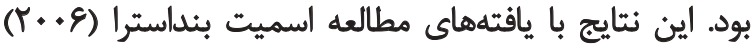

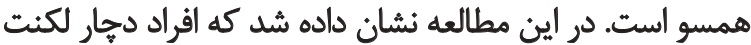

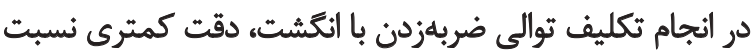

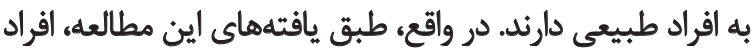

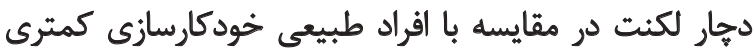

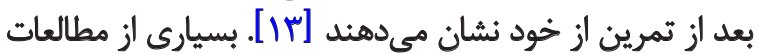

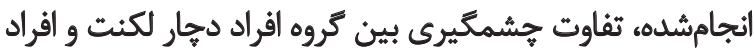

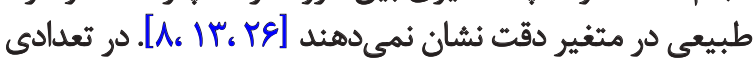

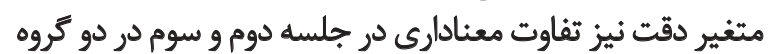

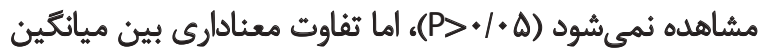

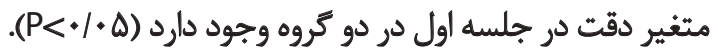

بs

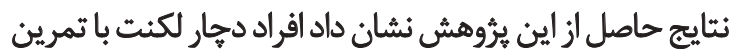

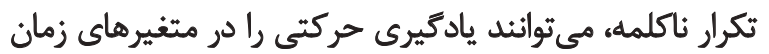

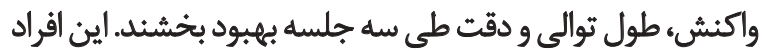

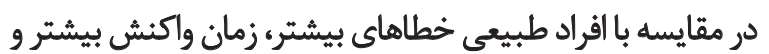

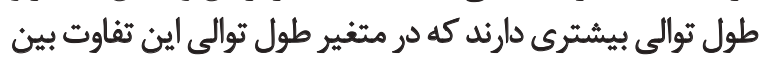

جدول Y. مقايسه ميانئين زمان واكتش، طول توالى و دقت بين كروه دجار لكنت و كروه طبيعى

\begin{tabular}{|c|c|c|c|}
\hline مقدار احتمال روز & مقدار احتمال روز r & مقدار احتمال روز 1 & متغيرهاي مريوط به يادكيرى حركتى \\
\hline .Mar & . Mar &.$/ r$ & زمان واكثش \\
\hline$\% r$ & $\% \infty$ & .1 .10 & طول توالى \\
\hline 吾 &.$/ N T$ &.$/ 4 \mathrm{r}$ & دقت \\
\hline
\end{tabular}


نشان دادند و اين تفاوت معنادار بود. اين يافته با نتايج مطالعه

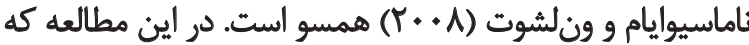

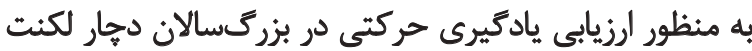

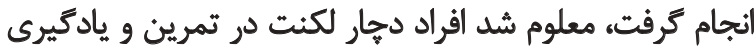

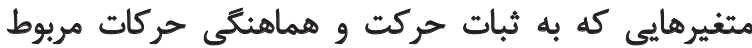

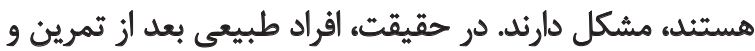

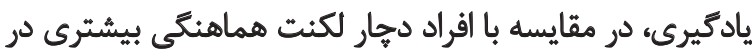

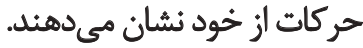

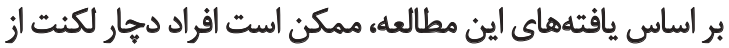

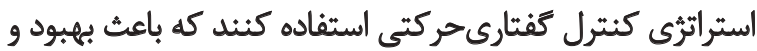

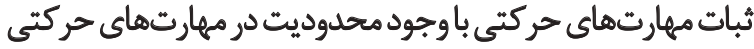

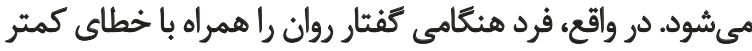

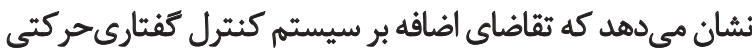

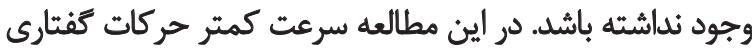

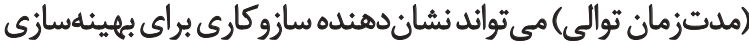

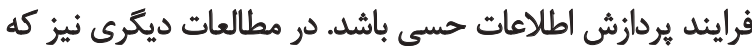

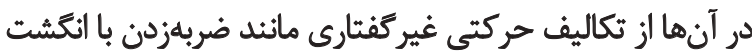

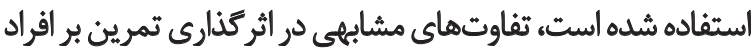
دجار لكنت و افراد طبيعى مشاهده شده است.

بنابراين، مى توان اين كونه نتيجه كرفت كه افراد دجار لكنت از

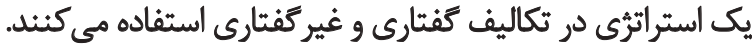

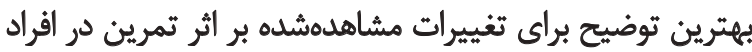

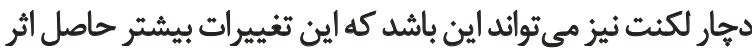

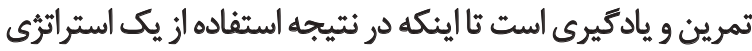

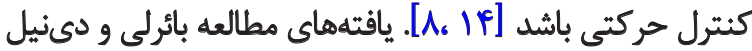

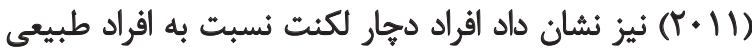

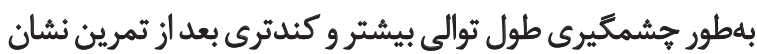

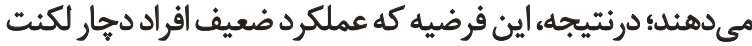
بعد از Tf ساعت دوره ثبات باقى مى ماند، ثأييد نشد.

يافتههاي اين مطالعه نشان داد طول توالى كندثر در افراد دجار

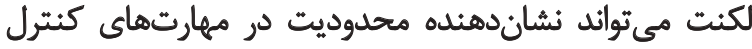

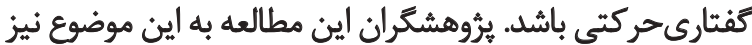

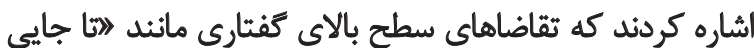

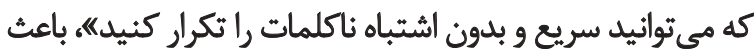

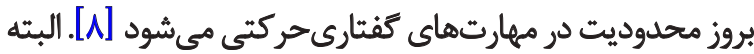

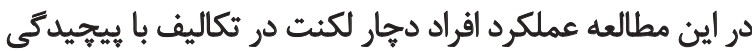

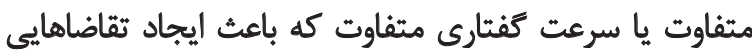

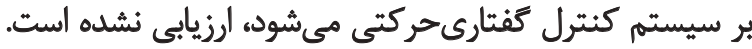
درنتيجه، اين موضوع در حد حدس بـ باقى مى ماندي

نتّايج اين مطالعه نيز با يافتههاي يُروهش حاضر همخوانى دارد. از مطالعات ديكرى كه نتايج آن با مطالعه حاضر همسو است،
ز مطالعات نيز تفاوت جشمخيرى بين گروه دجار لكنت و افراد

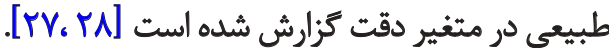

نتايج يرؤهش حاضر نشان داد در متغير دقت تفاوت جشمكيرى

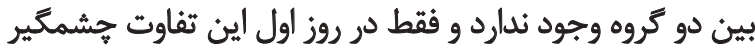

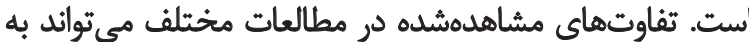

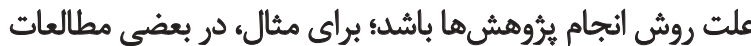

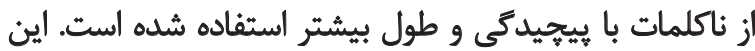

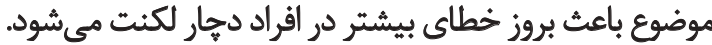
بر اساس يافتههاى اين مطالعه، افراد دجار لكنت مدتزمان

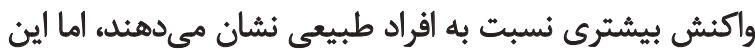

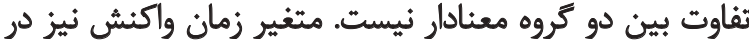

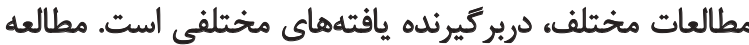

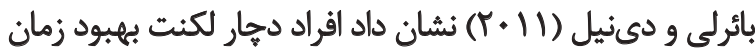

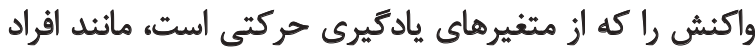

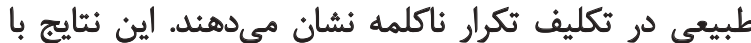

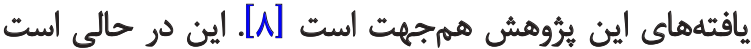

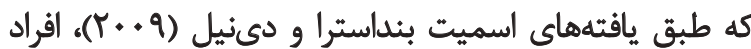

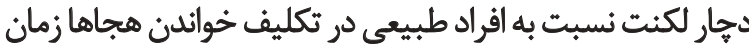

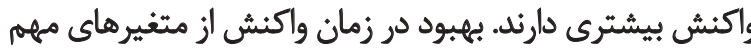

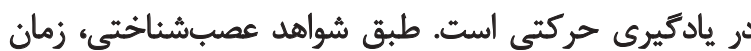

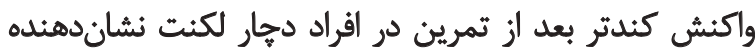

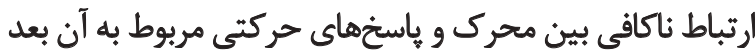

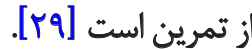

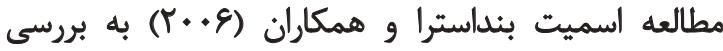

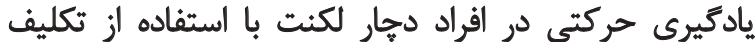

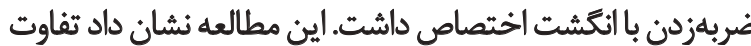

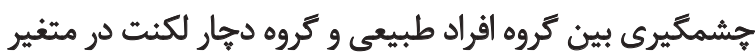

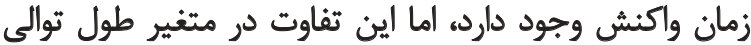

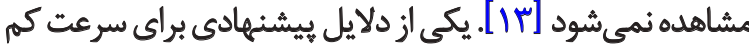

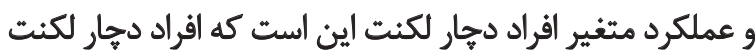

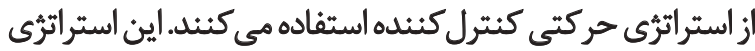

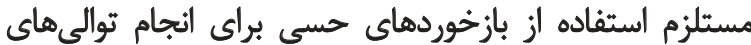

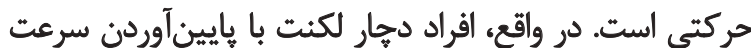

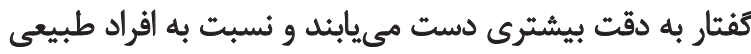

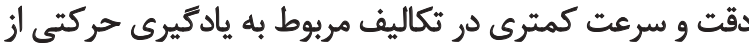

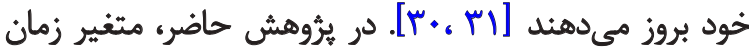

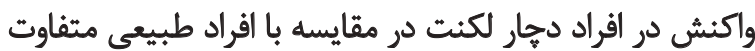

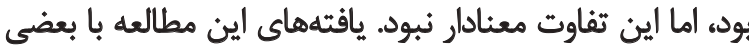

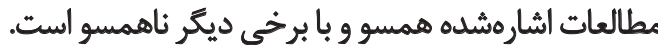
طبق يافتههاى يُؤوهش حاضر، افراد دجار الكنت مدتزمان

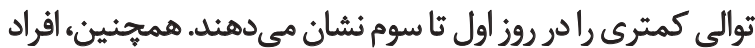
دجار لكنت ملتزمان توالى بيشترى را نسبت به افر الفراد طبيعى 
و برنامهاي درماني لكنت دارد، تعميميذيرى نتايج اندك است و

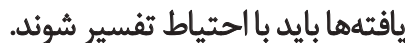

\section{تئيجنيرى}

طبق نتايج اين مطالعه، افراد دجار لكنته، بر اثر تمرين، شاهد

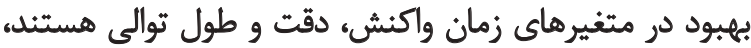

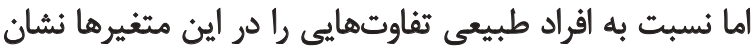

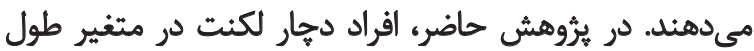

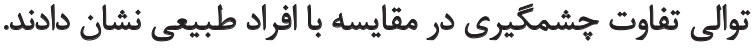

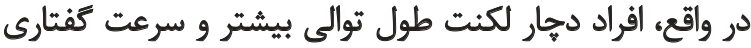
كمترى نسبت به افراد طبيعى نشان دادند. اين موضوع بر اساس كاس

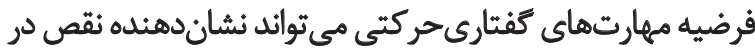

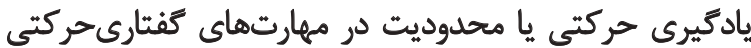

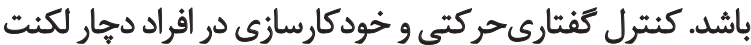

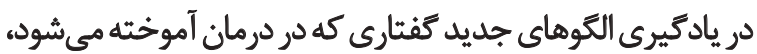

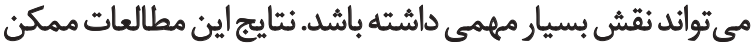

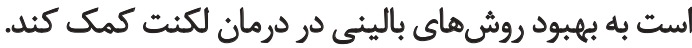
از محدوديتهاي اين بُوهش مي توان به دشوارى يافتن نمونهها

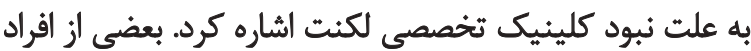

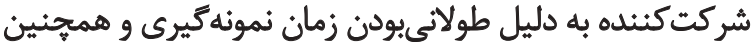

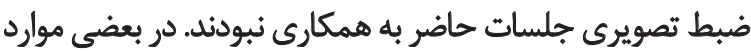

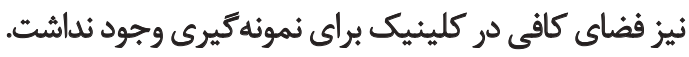

با توجه به اينكه اين مطالعات بيشتر روى بزركسالان دجار

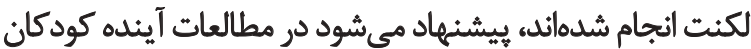

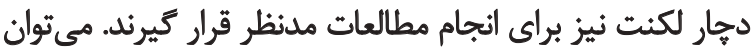

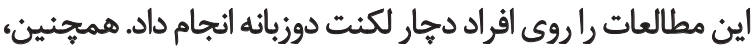

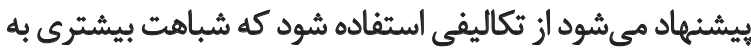

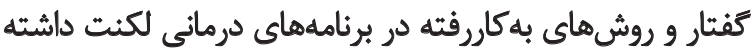

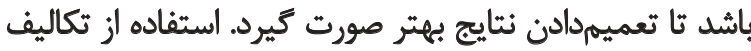

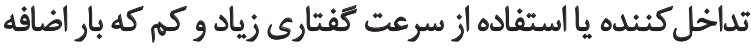

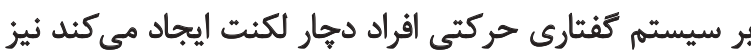

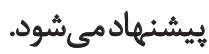

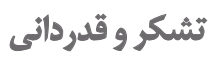

اين مقاله بخشى از يايان نامه مقطع كارشناسى ارشد نويسنده اول

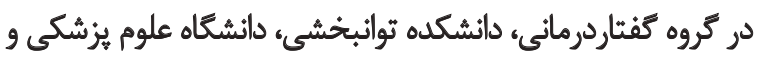

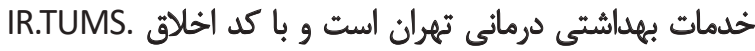

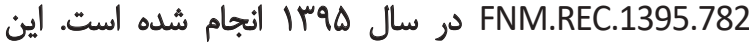

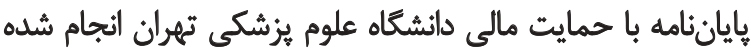

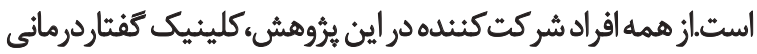

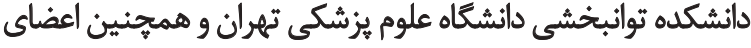

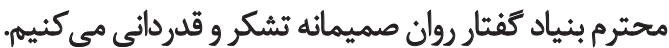

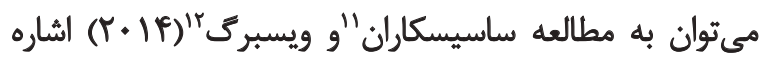

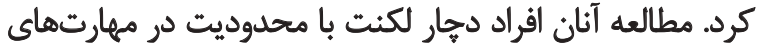

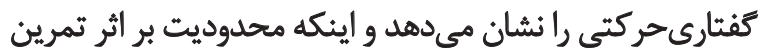

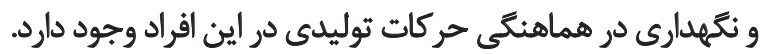

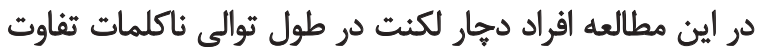
جشمكيرى را نسبت به افراد طبيعى نشان دادند. در اين مطالعه

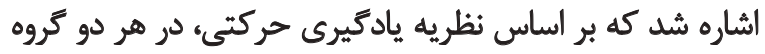

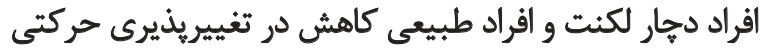

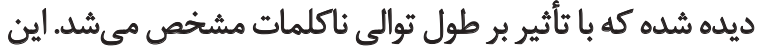

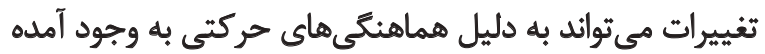

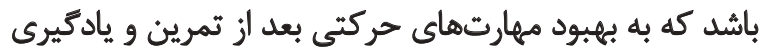

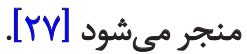
در فرضيه مهارتهاى كفتارىحركتى، مهارتهاى كفتارى

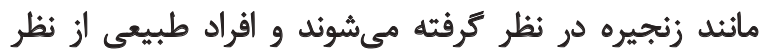

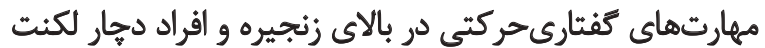

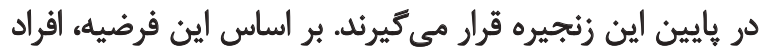

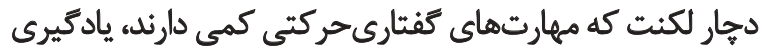

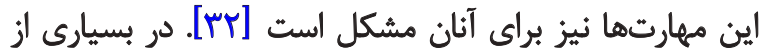

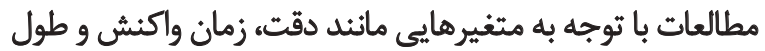

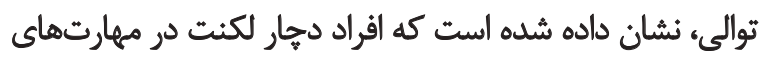

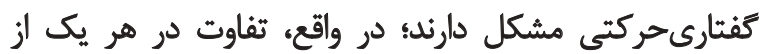

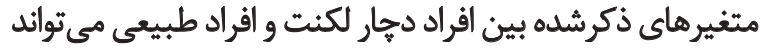

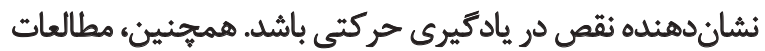

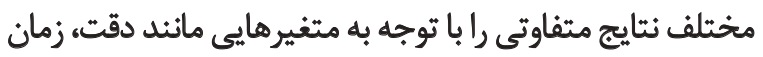

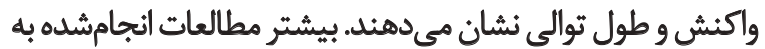

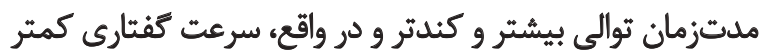

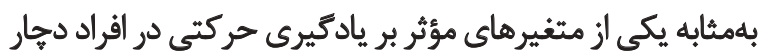
لكنت اشاره كرمانداند.

در بعضى مطالعات اشاره شده است كه افراد دجار لكنت توانايى

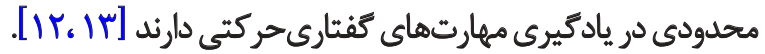

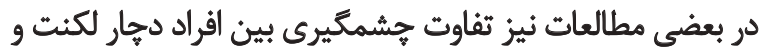

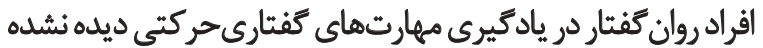

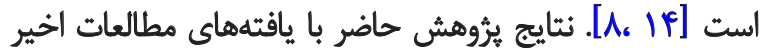

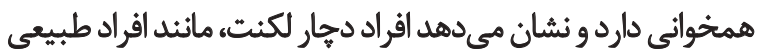

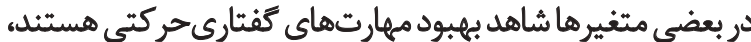

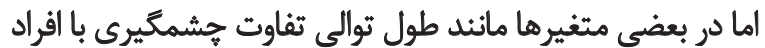

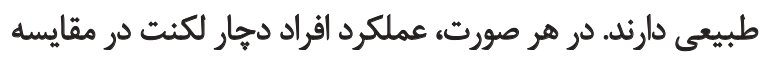

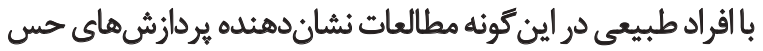

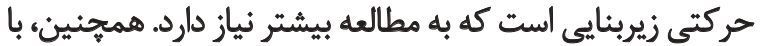

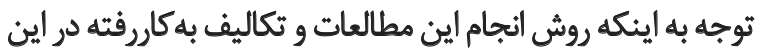

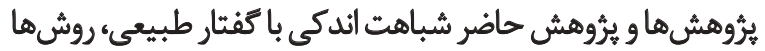

11. Sasisekaran

12. Weisberg 


\section{References}

[1] Guitar B. Stuttering: An intergrated approach to its nature and management. Baltimore, MD: Lippincott Williams \& Wilkins; 2006

[2] Heydari Nasrabadi M, Kamali M, Arrani Kashani Z. [Others' presence on the life experiences of people stuttering (Persian)]. Archives of Rehabilitation. 2015; 15(4):78-88.

[3] Fakar Gharamaleki F, Shahbodaghi MR, Jahan A, Jalayi S. Investigation of acoustic characteristics of speech motor control in children who stutter and children who do not stutter. Journal of Rehabilitation. 2016; 17(3):232-43. doi: 10.21859/jrehab-1703232

[4] Rahimi SS, Farazi M, Darouie A, Bakhshi E, Abdi S, Valinejad $\mathrm{V}$, et al. Comparison the quality of life among adults with and without stuttering: An emphasis on the severity of stuttering. Journal of Rehabilitation. 2016; 17(4):300-7. doi: 10.21859/jrehab-1704300

[5] Forster DC, Webster WG. Speech-motor control and interhemispheric relations in recovered and persistent stuttering. Developmental Neuropsychology. 2001; 19(2):125-45. doi: 10.1207/ s15326942dn1902_1

[6] Van Lieshout P, Hulstijn W, Peters HF. Searching the weak link in the speech production chain of people who stutter: a motor skill approach. In: Maassen B, Kent R, Peters H, van Lieshout P, Hulstijn W, editors. Speech Motor Control in Normal and Disordered Speech. Oxford: Oxford University Press; 2004.

[7] Abbruzzese G, Pelosin E, Marchese R. Current problems and strategies in motor rehabilitation for Parkinson's disease. Advances in Alzheimer's and Parkinson's Disease. 23-30. doi: 10.1007/978-0-387-72076-0_4

[8] Bauerly KR, De Nil LF. Speech sequence skill learning in adults who stutter. Journal of Fluency Disorders. 2011; 36(4):349-60. doi: 10.1016/j.jfludis.2011.05.002

[9] Namasivayam AK, van Lieshout P. Speech motor skill and stuttering. Journal of Motor Behavior. 2011; 43(6):477-89. doi: 10.1080/00222895.2011.628347

[10] Smits-Bandstra S, De Nil LF. Sequence skill learning in persons who stutter: Implications for cortico-striato-thalamo-cortical dysfunction. Journal of Fluency Disorders. 2007; 32(4):251-78. doi: 10.1016/j.jfludis.2007.06.001

[11] Tumanova V, Zebrowski PM, Goodman SS, Arenas RM. Motor practice effects and sensorimotor integration in adults who stutter: Evidence from visuomotor tracking performance. Journal of Fluency Disorders. 2015; 45:52-72. doi: 10.1016/j. jfludis.2015.04.001

[12] Webster WG. Response sequence organization and reproduction by stutterers. Neuropsychologia. 1986; 24(6):813-21. doi: 10.1016/0028-3932(86)90080-1

[13] Smits Bandstra S, De Nil L, Rochon E. The transition to increased automaticity during finger sequence learning in adult males who stutter. Journal of Fluency Disorders. 2006; 31(1):2242. doi: $10.1016 /$ j.jfludis.2005.11.004

[14] Namasivayam AK, van Lieshout P. Investigating speech motor practice and learning in people who stutter. Journal of Fluency Disorders. 2008; 33(1):32-51. doi: 10.1016/j.jfludis.2007.11.005
[15] De Nil LF. [Uncovering the neural basis of stuttering: Recen contributions from functional neuroimaging (Dutch)]. In E. Manders, D. Lembrechts, E. Bastijns editors. Stotteren. Recente inzichten. Leuven, Belgium: ACCO; 1999.

[16] Bloodstein O, Ratner B. A handbook on stuttering. Clifton Park, NJ: Thomson Delmar Learning; 2008

[17] Smits Bandstra S, De Nil LF, Saint Cyr JA. Speech and nonspeech sequence skill learning in adults who stutter. Journal of Fluency Disorders. 2006; 31(2):116-36. doi: 10.1016/j.jfludis.2006.04.003

[18] Fitzgerald HE, Cooke PA, Greiner JR. Speech and bi manual hand organization in adult stutterers and nonstutterers. Journal of Fluency Disorders. 1984; 9(1):51-65. doi: $10.1016 / 0094-730 x(84) 90007-x$

[19] Wingate ME. Stuttering: Theory and treatmen. Hoboken, NJ: John Wiley \& Sons Inc; 1976.

[20] Sayyahi F, Soleymani Z, Mahmoudi Bakhtiyari B, Jalaie S. Providing a non word repetition test in 4-year-old Persian children and determining its validity and reliability. Bimonthly AudiologyTehran University of Medical Sciences. 2011; 20(2):47-53.

[21] Afshar MR, Ghorbani A, Jalilevand N, Kamali M. Providing the non-word repetition test and determining its validity and reliability and comparing phonological working memory in 4 to 6 Farsi-speaking normal and SSD children in Tehran City. Journal of Research in Rehabilitation Sciences. 2013; 9(5):899-911.

[22] Ludlow CL, Siren K, Zikria M. Speech production learning in adults with chronic developmental stuttering. In: Hulstijn W, Peters HF, van Lieshout PH, editors. Speech Production: Motor Control, Brain Research and Fluency Disorders. Amsterdam: Elsevier; 1997.

[23] Alfonso PJ, Van Lieshout PH. Spatial and temporal variability in obstruent gestural specification by stutterers and controls: Comparisons across sessions. In: Hulstijn W, Peters HF, van Lieshout PH, editors. Speech production: Motor control, brain research and fluency disorders. Amsterdam: Elsevier; 1996.

[24] Schulz GM, Dingwall WO, Ludlow CL. Speech and oral motor learning in individuals with cerebellar atrophy. Journal of Speech Language and Hearing Research. 1999; 42(5):1157. doi: 10.1044/ jslhr.4205.1157

[25] Pytel JL. The relation of kinematic factors to the acquisition of skill on a novel task. Canadian Journal of Applied Sport Sciences. 1980; 5(1):44-8. PMID: 7389047

[26] Smith A, Sadagopan N, Walsh B, Weber Fox C. Increasing phonological complexity reveals heightened instability in inter-articulatory coordination in adults who stutter. Journal of Fluency Disorders. 2010; 35(1):1-18. doi: 10.1016/j.jfludis.2009.12.001

[27] Sasisekaran J, Weisberg S. Practice and retention of nonwords in adults who stutter. Journal of Fluency Disorders. 2014; 41:55-71. doi: 10.1016/j.jfludis.2014.02.004

[28] Ludlow CL, Siren K, Zikria M, Hulstijn W, Peters HF, van Lieshout PH. Speech production learning in adults with chronic developmental stuttering. In: Hulstijn W, Peters HF, van Lieshout $\mathrm{PH}$, editors. Speech production: Motor control, brain research and fluency disorders. New York: Springer; 1997. 
[29] Smits Bandstra S, De Nil L. Speech skill learning of persons who stutter and fluent speakers under single and dual task conditions. Clinical Linguistics \& Phonetics. 2009; 23(1):38-57. doi: 10.1080/02699200802394914

[30] Van Lieshout PHHM, Hulstijn W, Peters HFM. From planning to articulation in speech production: What differentiates a person who stutters from a person who does not stutter. Journal of Speech Language and Hearing Research. 1996; 39(3):546. doi: 10.1044/jshr.3903.546

[31] Fitts PM. The information capacity of the human motor system in controlling the amplitude of movement. Journal of Experimental Psychology. 1954; 47(6):381-91. doi: 10.1037/h0055392

[32] Van Lieshout P, Hulstijn W, Peters HF. Searching the weak link in the speech production chain of people who stutter: a motor skill approach. In: Maassen B, Kent R, Peters H, van Lieshout P, Hulstijn W, editors. Speech Motor Control in Normal and Disordered Speech. Oxford: Oxford University Press; 2004. 